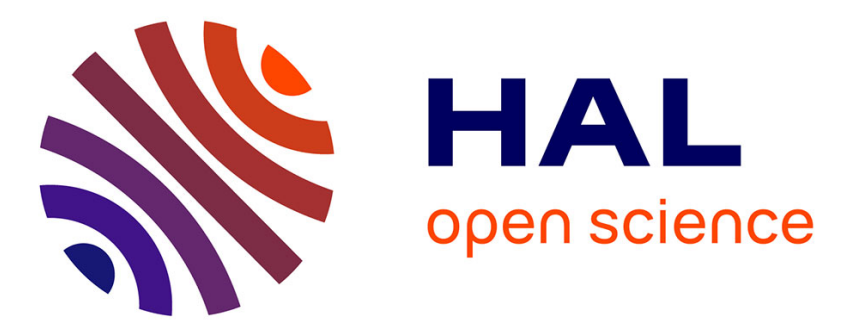

\title{
Experimental Analysis and Characterization of a Wireless Sensor Network Environment
}

\author{
Bogdan Pavkovic, Fabrice Theoleyre, Andrzej Duda
}

\section{To cite this version:}

Bogdan Pavkovic, Fabrice Theoleyre, Andrzej Duda. Experimental Analysis and Characterization of a Wireless Sensor Network Environment. PE-WASUN 2010 - International Symposium on Performance Evaluation of Wireless Ad Hoc, Sensor, and Ubiquitous Networks, 2010, Bodrum, Turkey. pp.25, 10.1145/1868589.1868595. hal-01073416

\section{HAL Id: hal-01073416 https://hal.inria.fr/hal-01073416}

Submitted on 2 Jun 2020

HAL is a multi-disciplinary open access archive for the deposit and dissemination of scientific research documents, whether they are published or not. The documents may come from teaching and research institutions in France or abroad, or from public or private research centers.
L'archive ouverte pluridisciplinaire HAL, est destinée au dépôt et à la diffusion de documents scientifiques de niveau recherche, publiés ou non, émanant des établissements d'enseignement et de recherche français ou étrangers, des laboratoires publics ou privés. 


\section{Experimental Analysis and Characterization of a Wireless Sensor Network Environment}

\author{
Bogdan Pavkovic \\ Grenoble Informatics \\ Laboratory (LIG) \\ University of Grenoble \\ 681 , rue de la Passerelle \\ F38402 Saint Martin d'Heres \\ pavkovic@imag.fr
}

\author{
Fabrice Theoleyre \\ CNRS, LSIIT \\ University of Strasbourg \\ Bd Sebastien Brant \\ F67402 Illkirch \\ theoleyre@unistra.fr
}

\author{
Andrzej Duda \\ Grenoble Informatics \\ Laboratory (LIG) \\ University of Grenoble \\ 681 , rue de la Passerelle \\ F38402 Saint Martin d'Heres \\ duda@imag.fr
}

\begin{abstract}
Existing testbeds, even though rare and specialized, are not used to their full potential. Collected data during an experimentation is just used to evaluate some specific tested aspect. To further benefit from the knowledge gathered on a testbed and to obtain the insight into the WSN environment itself, we propose a thorough statistical analysis. Some of our analysis include radio link characterization, its correlation with environmental parameters as well as an insight into network dynamics from the point of view of a node and a link. We also discuss how testbeds should be designed or improved to provide more detailed information necessary for an advanced analysis.
\end{abstract}

\section{Categories and Subject Descriptors}

C.2.1 [Computer-Communication Networks]: [Network Architecture and Design - Wireless Communication]

\section{Keywords}

wireless sensor networks, testbeds, characterization, statistical analysis

\section{INTRODUCTION}

Wireless Sensor Networks have attracted a lot of attention for a few years. Most of the research effort has focused on the two main issues: routing [1] and power-energy savings [2]. However, the research community has become aware that models of wireless multihop networks are too simplistic and lead to misleading conclusions. In particular, different simulators have been proved to provide different results [3]. The radio model has especially a strong impact on performance [4].

To advance the evaluation of various protocols, we can setup an ad hoc testbed to compare the simulation results with measurements gathered on the testbed. However, the mea-

Permission to make digital or hard copies of all or part of this work for personal or classroom use is granted without fee provided that copies are not made or distributed for profit or commercial advantage and that copies bear this notice and the full citation on the first page. To copy otherwise, to republish, to post on servers or to redistribute to lists, requires prior specific permission and/or a fee.

PE-WASUN'10 October, 17-21, 2010, Bodrum, Turkey

Copyright 20XX ACM X-XXXXX-XX-X/XX/XX ...\$10.00. surements usually concern only a limited number of tested aspects and setting up operational testbeds requires important human effort.

Developing new testbeds to accelerate prototyping and to make their evaluation easier has become an interesting research objective. Orbitlab [5] proposed for example to deploy a testbed in a dynamically reconfigurable grid. Other authors provide guidelines to design feasible protocols: they prone the principle of the simplest is the best [6]. However, these desirable efforts usually do not provide generic results to the networking research community, for instance they do not consider many important aspects such as: What are the characteristics of the WSN radio topology? What is the reliability of a WSN? Are the properties stable or do they exhibit some variability or periodicity? We propose here to address one part of these fundamental concerns.

In the past, the statistical analysis was applied to traffic analysis [7] or anomaly detection [8] to extract some correlations and salient features. We propose in this paper to use this mathematical approach to characterize the performances and properties of a WSN. Our analysis includes in particular:

- characterization of radio links in a WSN: their reliability and the correlation between their properties;

- the analysis of the network dynamics: how does a WSN change in time?

- how can we predict the quality of a radio link with a local and simple measure?

- how can we discard measurement errors?

\section{TESTBED DESCRIPTION}

We used a testbed originally designed for validating a routing protocol [9]. It was composed of 36 Coronis nodes implementing the Wavenis technology [10]: a fast frequency hopping is implemented to be robust to narrow band noise. The MAC layer follows a CSMA-CA approach for medium access contention. Besides, two nodes acted as sinks with a direct connection to the Internet and a database for storing received packets. Nodes were deployed over the area of the technical park of Orange Labs in Meylan, France, both indoor and outdoor. We analyzed the measurements of 18 days of operation. Figure 1 presents the deployed topology in the urban environment. 


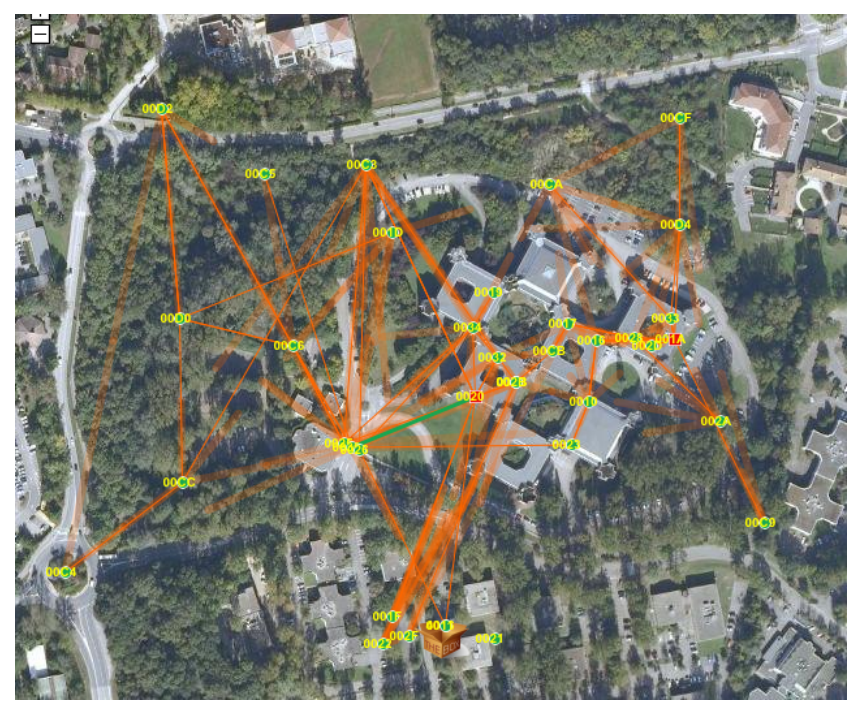

Figure 1: Deployed topology in an urban environment

The testbed was mainly used to validate a routing protocol based on virtual coordinates: each node maintains a metric related to its virtual distance to the sink [11]. The next hop is chosen as the neighbor that is virtually the closest to the sink.

Nodes periodically perform a neighborhood discovery every 13 minutes and maintain a proactive neighborhood table including the virtual distance and RSSI of each neighbor.

Each node generates a new data packet every 17 minutes. This packet is transmitted in anycast: any sink can be used to reach the wired part of the network. In order to find the next hop (the node that has the lowest virtual distance), current node has just to walk in its neighborhood table.

The routing packets, aside from control fields (source and destination ID, sequence number, etc.), contain complete neighborhood tables (the neighbor ID and the received RSSI value (32 possible levels between $-108 \mathrm{dBm}$ and $-60 \mathrm{dBm}$ with a step of $1.5 \mathrm{dBm}$ ) and the measurements of the temperature, humidity, and light sensors at the instant just before sending the packet. Packets successfully received at sink nodes were labeled with a timestamp and stored in a database. Table 1 sums-up the important testbed information.

\section{METHODOLOGY}

\subsection{Database description}

To allow meaningful interpretation and easy use of different types of measured values contained in the received routing packets, the database is divided into few tables:

- the node ID and its geographical position to obtain the geographical topology. We can compare it to the radio topology;

- neighborhood information (neighbor ID and a RSSI value). We can observe in particular duration and quality of the links;

- sensor measurements (e.g. humidity, temperature).

\begin{tabular}{|c||c|}
\hline \hline Environment type & Urban \\
\hline Node position & Indoor \& outdoor \\
\hline Sensor type & Coronis Wavenis \\
\hline Number of nodes (sinks) & $36(2)$ \\
\hline Duration of the experiment & 18 days \\
\hline Neigh. discovery period & 13 min. \\
\hline Data packet generation period & 17 min. \\
\hline
\end{tabular}

Table 1: Testbed parameters

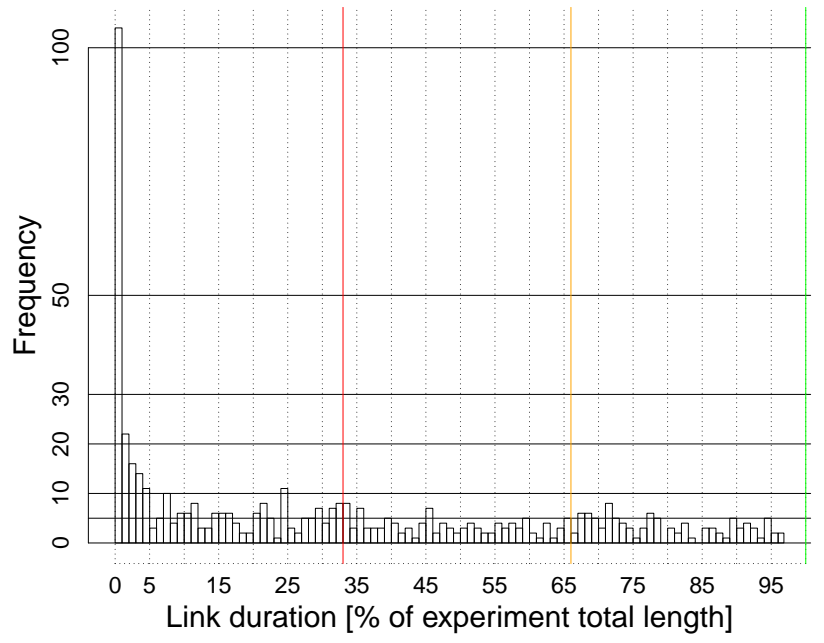

Figure 2: Distribution of cumulative link duration

On the average, each node sent 1,500 data packets (maximum sample size) to sinks, where just the ones successfully arrived were saved in the database. To perform an accurate statistical analysis, we need to discard received data samples with insufficient cardinality. Thus, we have removed all the data samples that count less than $1 \%$ of the maximum size (i.e. 15 entries).

\subsection{Bidirectional and Unidirectional links}

We can distinguish between unidirectional and bidirectional links (RSSI measures are available for one or for both directions). We obtained 16 unidirectional links and 280 bidirectional links.

We define cumulative link duration as ratio between number of node appearances in the neighborhood table and total number of tables. In other words it represent percent of the cases where link between two nodes was detected and qualified with RSSI value. We can notice in the Figure 2 that a significant number of links (20\%) last less than $1 \%$ threshold. By filtering these sets with too small cardinality we eliminated in particular all of the unidirectional links: their data sets counted only between 1 to 4 measures. Thus, one of our first results is that the testbed did not have any unidirectional links. However, some of the bidirectional links can be asymmetrical (i.e. their quality is different for both directions), as explained in one of the following section.

Unidirectional links may appear when antennas are not perfectly omnidirectional [12], the filters are not well-conceived [13] or when the nodes do not use the same transmission power [14]. Consequently, we can conclude that Wave- 
nis nodes are robust and the hardware is well conceived and industrialized (i.e. different nodes have the same characteristics).

\subsection{Filtering data}

Since we focus on experimental data, we have to discard ambiguous measures (i.e. possible outliers or impracticable values) to obtain unbiased results. We propose to detect and discard this kind of values.

Formally, we consider that a value is an outlier, if it conforms to the following condition:

$$
x<Q 1-1.5 \cdot I Q R \quad \vee \quad x>Q 3+1.5 \cdot I Q R
$$

where Q1 represents first quantile of observed data set, Q3 third quantile and IQR difference between them i.e. interquantile range.

Thus, we discard all the values that are single isolated outliers: only one value is extreme, corresponding surely to a transient behavior. On the contrary, multiple consecutive outliers could arise from temporary obstacles (e.g. a delivery truck, a car) for radio propagation, climatological changes (raining that disturbs radio transmissions and increases the humidity measures). Thus, we keep all multiple consecutive outliers. In other words, we consider that the extreme values that last for more than 17 minutes are valid. We will give more attention to multiple consecutive outliers later in the article to infer the main causes and consequences.

After filtering our experimental dataset, we proceed with the analysis.

\section{LINK QUALITY}

The progress in the radio chip design positively impacted the performance and reliability of WSNs [15]. This motivated us to further investigate the possibility to use the RSSI value as a reliable link quality indicator.

\subsection{Radio link Symmetry}

We measured the RSSI value in both directions for each radio link (Figure 3). We did not remove in this graph the links with a very small number of values (as explained in section 3.3) because we aim here at analyzing the reason of their existence.

When the points are close to the diagonal, the links are symmetrical: the quality is identical in both directions. The reader can remark that contrary to the literature, symmetry is predominant.

This means that nodes use the same transmission power. Besides, they are also homogenous: the radio hardware behaves identically. For instance, the radio chips of two different radio modules follow the same frequency selectivity (i.e. filters are identical).

Radio links are seldom asymmetrical: these outliers appear for links with the duration less than $1 \%$ of total length of the experiment. For these rare cases, the quality in one direction is significantly different, i.e. greater than $10 \%$, sometimes even $55 \%$. This unbalanced representation justifies the removing of links with too small cardinalities.

\subsection{RSSI distribution}

To predict the link behavior with a local and simple metric, the measure should follow a known probability distribution model: we would be able to accurately infer the average

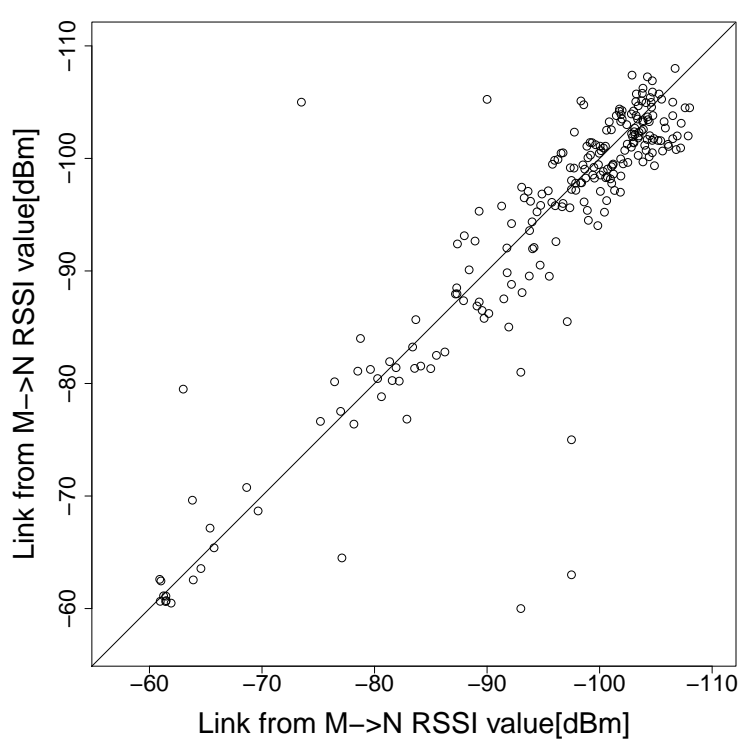

Figure 3: Symmetry of existing bidirectional links

quality of the link by analyzing in real-time the measured values.

The Normal (or gaussian) distribution is extensively used since it models well many natural phenomenons, especially for radio propagation (e.g. the Additive White Gaussian Noise). We aim here at verifying if the RSSI measured for each of the existing links follows this distribution.

We use the Shapiro-Wilk test [16], applied to the measured RSSI. For $92 \%$ of the links, the p-value of the Shapiro-Wilk test was significantly less than 0.05 and for the rest barely over this value. This signifies that we need to reject the null-hypothesis, meaning that the RSSI does not follow a normal distribution. This corroborates some indoor results [17] and even in outdoor conditions for LOS radio links, the RSSI does not follow a normal distribution.

We compared also these RSSI samples to other two wellknown distributions: Logistic and Cauchy. We used a Kolmogorov-Smirnov test, permitting to compare a well-known distribution to a collection of samples. More precisely, a collection of values are generated according to the well-known distribution, with the same cardinality as the set we want to compare to. Then, the Kolmogorov-Smirnov test let us know if the two collection of values follow the same distribution. For both distribution cases and for all of the links resulting p-value was always close to 0 , meaning that we have to drop null-hypothesis i.e. RSSI samples do not follow neither Logistic neither Cauchy distribution. Nevertheless, RSSI distribution that we tried to describe, has bell shape with high central peak but it is little bit skewed on one side (Figure 4). Thus, no well-known distribution permits to have a generic model for such RSSI values.

We now aim at demonstrating that the RSSI of the different links follow the same distribution. Since they do not follow the Normal distribution we have chosen one of the most familiar non-parametric test- the Wilcoxon-Mann-Whitney 


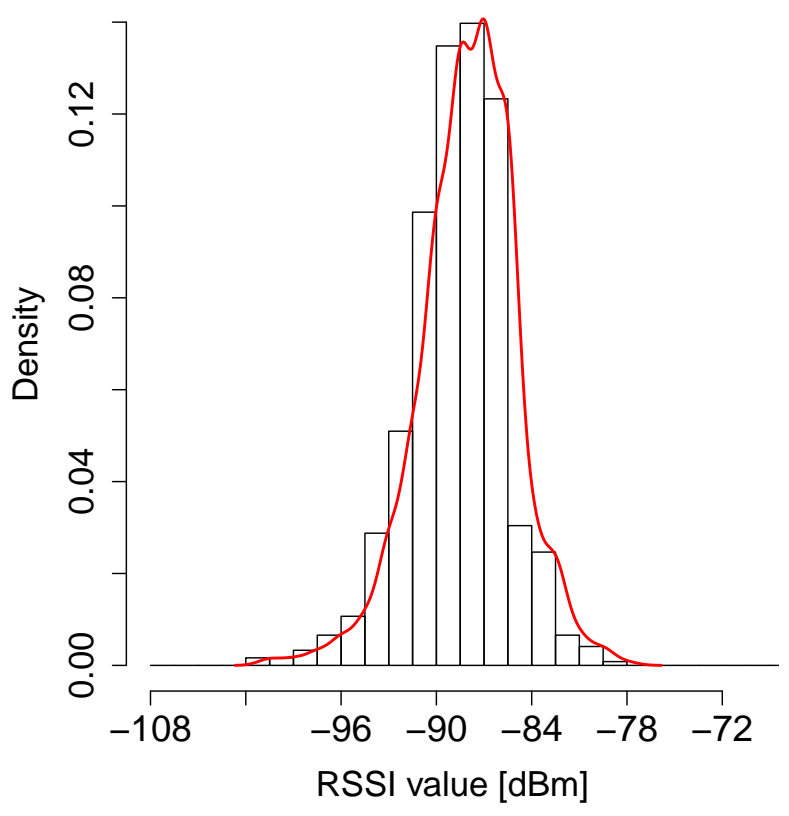

Figure 4: Distribution of RSSI value for one of the representative link

Test [16]. Now the null-hypothesis is that samples from two independent samples come from the same distribution. The average value of p-value for this test was 0.0416 while $89 \%$ of values were smaller than 0.05 . The null-hypothesis is valid since this p-value is lower than alpha level 0.05. We conclude in other words that the corresponding pairs of samples do follow the same distribution.

This can be also observed in Figure 5. We plotted the Box Plots for some representative links. Even though median values are not perfectly aligned, we can notice that inter quantile ranges are similar as well as the skewness of data and $\max / \min$ values.

\subsection{RSSI periodicity}

We also analyzed the difference in radio link quality during working hours (8am-7pm) and night periods (9pm-6am). Plotting the values we obtained almost the same graph as the one plotted in Figure 3 showing that links did not change their properties during different periods of day. Thus, movements of people and vehicles in the technical park during working hours do not have any significant impact on the RSSI. RSSI is stable and transmissions are quite robust to some environment properties changes.

In other words, the MAC and PHY layers in wavenis are robust to noise and environment changes. We conjecture that the fast frequency hopping (a packet is transmitted through several frequencies) and CSMA-CA help to achieve such a feature.

\subsection{RSSI vs. Cumulative link duration}

To have a more detailed insight into the cumulative link duration property shown in the Figure 2, we tried to observe whether it can be correlated with the RSSI value.

Figure 6 shows Box Plots for all recognized links in the testbed separated in 4 groups according to the range of their

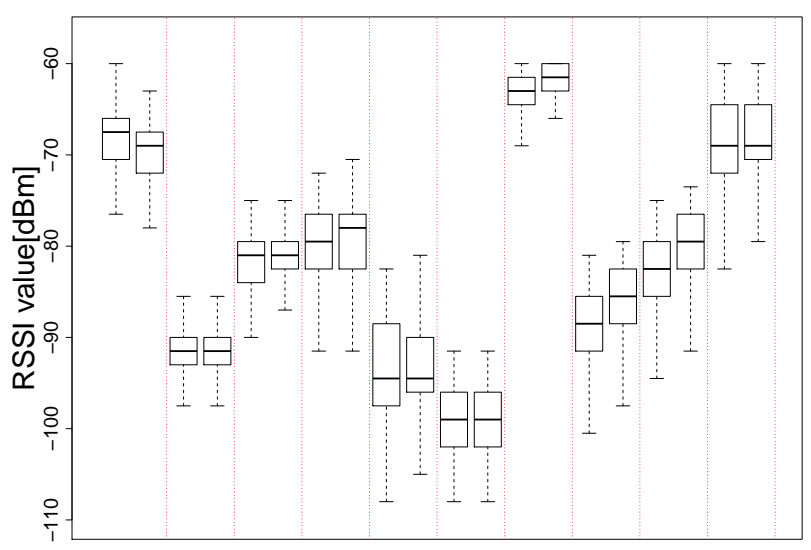

Figure 5: Box plot showing that bidirectional links follow the same distribution (with similar parameter's values

cumulative duration without sorting them in ascending order by same criteria.

Looking at this figure we can notice that there is no evident correlation between RSSI value and cumulative link duration since Box Plot of RSSI covers whole extent of possible values in different link cumulative duration ranges. However, we can remark the following points:

- a single RSSI distribution for a particular link does not permit to conclude on the duration for this link. Individual conclusions are not possible;

- if we take a closer look on the graph, we can remark that each category exhibits different RSSI spreadings. In other words, we could derive a probability of duration for different RSSI values. However, this constitutes a global (and not individual) behavior, i.e. RSSI is not directly a good quality estimator;

- for the first range of cumulative link duration (1-40\%), mean value of the RSSI for all of the links do not pass above $-90 \mathrm{dBm}$. Thus, a poor link means obligatory a low RSSI;

- the largest RSSI values mean in most cases we face to stable links.

\subsection{RSSI vs. sensor measurements}

For a long time the research and radio chip developer community believed that high humidity can be considered as one of the main causes of the decrease in the quality of radio communication.

First, we verified the correlation (linear dependence) between the measured humidity and the RSSI. We computed the Pearson's correlation factors [16] for all bidirectional links. In all the cases, the value did not exceed 0.5 , plus we have neither a negative nor a positive correlation between the two variables. In the same way, the correlation is not significant if we focus only on outdoor radio links.

The second test was an attempt to further explore the correlation between humidity and RSSI values, but just taking into account the impact of the extreme (maximum) values of humidity measurements. We extracted subset of top $25 \%$ of 


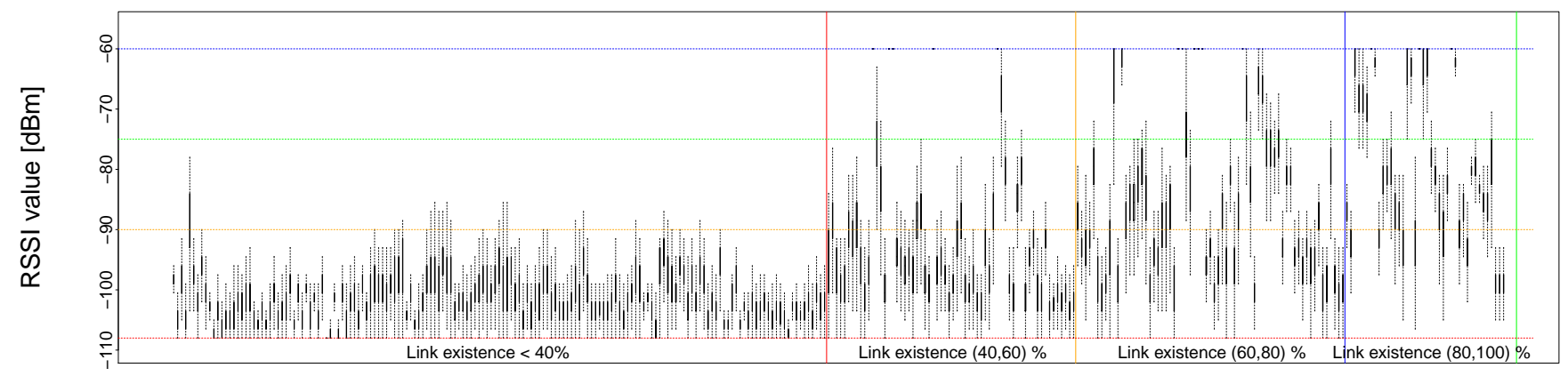

Figure 6: Impact of the RSSI value on link existence

all humidity values measured during the experiment. Afterward, we computed the difference between the mean value of RSSI for the observed link and the current value of RSSI at the same temporary instant as the measured extreme value of humidity.

If the humidity does not impact the RSSI, we would have an average difference equals to 0 . This means that the RSSI value has the same chance to be greater or lower than the average RSSI value. Since we observed this behavior (values are very closed to 0 , with a varying sign), we can for sure say that humidity has no impact on the RSSI measurements.

In conclusion, Coronis radio chip is very robust. In particular, Wavenis uses a fast frequency hopping approach to avoid interferences and frequency-selective fading.

\section{NETWORK DYNAMICS}

The radio channel is intrinsically unstable, since it is easily influenced by various environmental parameters. This creates a certain dynamism in the network, where links can easily disappear or re-appear. To optimize the performances, the deployed MAC, topology control and routing protocols should self-adapt to changes. We will now focus on the network dynamics to understand how it could further impact the higher layers.

\subsection{Neighborhood variation}

We first studied the variation in the neighborhood table. The same remarks hold for all the nodes, and we focus here on one node, selected randomly. We plotted in Figure 7 the variation of its neighborhood size.

It varies most of the time with rare stable periods that last at most few samples. We have recognized this behavior as a general trend for all the nodes. This raises the question of whether a proactive approach is the most accurate solution for discovering its neighbors. Indeed, proactive maintenance may result in inefficient routing decisions when choosing non reliable nodes: they can be chosen as next hop because an hello was previously received although they will not receive correctly the next data packet. This result tends to conclude that opportunistic solutions, where the next hop is chosen only when the data packet is transmitted, is more relevant in this environment. Since the next hop is chosen reactively among the nodes that received the data packet, unreliability problem is reduced.

\subsection{Link's Evolution}

Although the neighborhood table changes continuously, a group of stable neighbors may practically exist. In particular, can a large neighbor's stability be correlated with e.g. the RSSI or the distance between the transmitter and the receiver?

We have noticed that stable radio links have one of the following properties:

- an high RSSI value (superior to $-75 \mathrm{dBm}$ );

- a pair of nodes closer one fifth of the radio range $(\approx 70 \mathrm{~m})$ and having a medium value of RSSI (between $-75 \mathrm{dBm}$ and $-90 \mathrm{dBm})$.

By combining distance and RSSI information, we should be able to predict the link stability. Moreover, there was no single case in which neighbors with a high value of RSSI were not among most stable neighbors.

High RSSI could be used as reliable indicator of link stability when using Wavenis chips [10]. Geographical information is a plus to cope with medium RSSI values. Similar observation was made by other authors [15] for different type of radio chips. Nevertheless, there is still substantial free space in order to make tighter conclusions about the link behavior with RSSI in a gray zone (low levels close to the threshold) since it is influenced by various effects (multipath, fading, interference, etc.) for which the impact varies during the time and according to the situation.

\subsection{Multiple consecutive outlier distribution}

As previously stated in section 3.3 we have kept multiple consecutive outliers since they depict transitory effect that influence the quality of radio channel for a short period.

We aim here at analyzing this phenomenon in a more global way, i.e. for all observed links in the network. Thus, we extracted the Cumulative distribution function (CDF) of the number of multiple consecutive outliers. We plotted the results in the Figure 8.

In particular, we have 4 or less consecutive outliers in $75 \%$ of the cases (the blue circle in Figure 8). In the same way, for $90 \%$ of the cases, we have 8 or less multiple consecutive outliers (dashed line in the figure). This means that for most of the cases, we can consider multiple consecutive outliers lasting up to 8 periods as transitory effects which interrupt stable radio link for short period. On the contrary, longer outliers means that the radio link has probably changed definitively, because e.g. the node was moved or the environment changed (e.g. building modifications).

Let still focus on the 4 consecutive outliers case. We have approximately $12 \%$ of the samples that last for exactly 4 


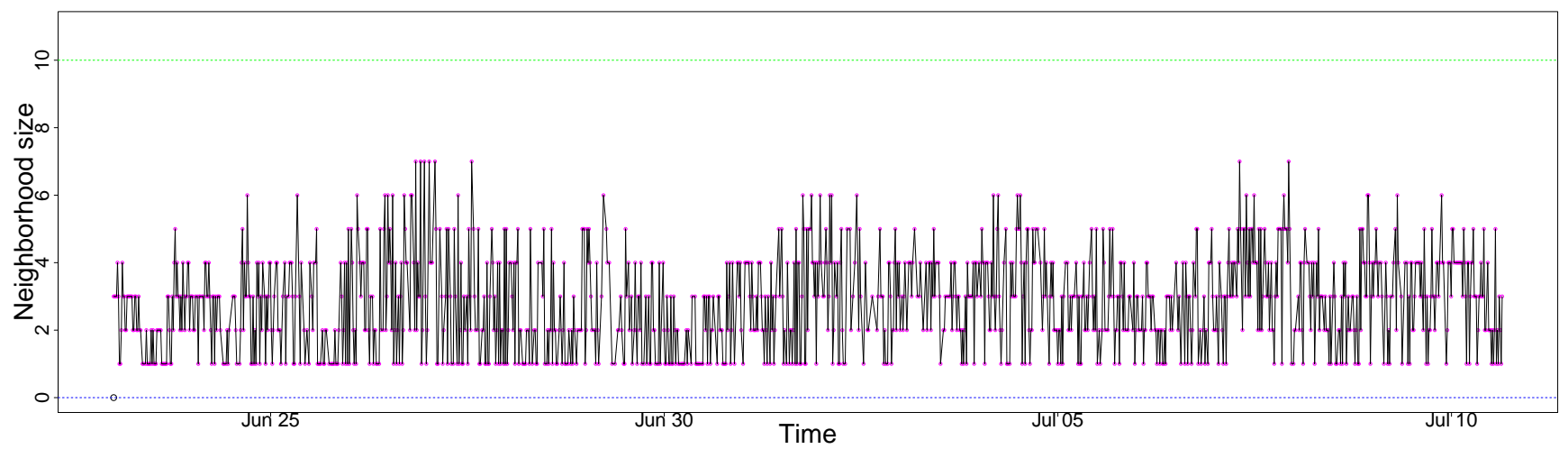

Figure 7: Variation in the neighborhood size for one of the nodes

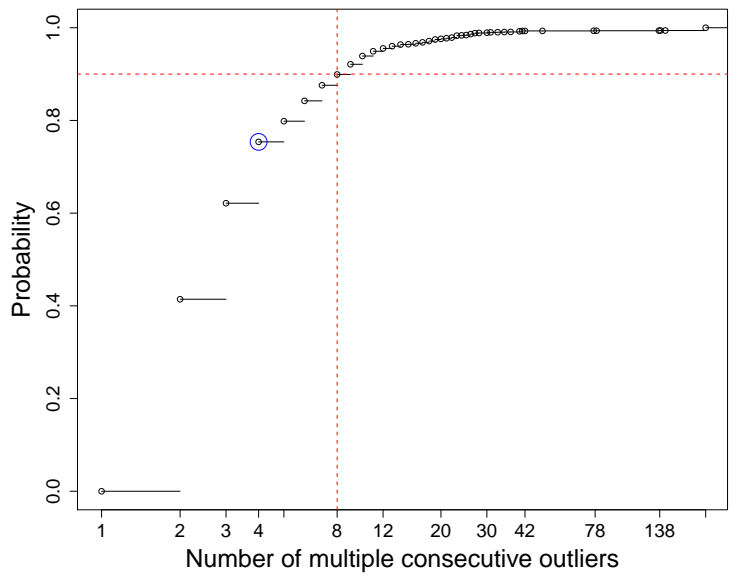

Figure 8: CDF of multiple consecutive outlier

outliers $(75 \%-63 \%)$ ), and only $5 \%$ of th samples that last for exactly 5 outliers $(80 \%-75 \%)$. In other words, when a node experiences 4 consecutive outliers, it has a larger chance that the next sample will be normal than it will be still an outlier. We can remark that this observation holds for all the cases: we have a strictly larger probability to have $\mathrm{k}$ consecutive outliers than $\mathrm{k}+1$. In other words, outliers have a limited impact and the average and mean values would be wellestimated if they have properly detected and discarded (i.e. they will not introduce a large bias).

\subsection{Number of outliers in a sliding window}

Problem occurs when we want to detect outliers practically. It is almost impossible to keep the whole history in node's memory in order to precisely calculate IQR and to remove right outliers. To cope with this problem, we may only save the last samples. We chose here to implement a sliding window of 20 samples. Further, we have calculated the Cumulative Distribution Function of the number of outliers per sliding window (Figure 9) to justify our choice.

In $97.5 \%$ of cases, we have 4 or less outliers per sliding window (cf. the dashed line). We aim here at limiting the memory consumption while keeping on well-estimating

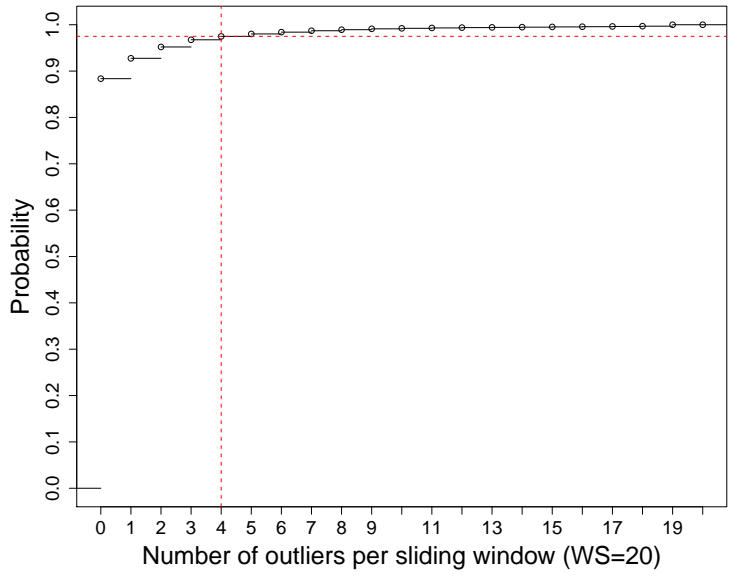

Figure 9: CDF of number of outliers per sliding window of size 20 samples

the average value to be able to detect right outlier values. Moreover, the method must not be too conservative since testbeds are not static and the environment can change. In particular, we should not consider all the new measures as outliers in this situation: the quality should sometimes be re-estimated, even if new values are far from the previous average value.

We propose following approach to reach this objective. At most 4 slots will be used to store outlier values (yellow fields in Figure 10). These values will be flagged and won't be used to calculate the IQR value (eq. 1) since we consider that these values are abnormal. Eventually, a new value could be detected as outlier although 4 values were already flagged outliers. In this case, we un-flag the outlier closer to the median value. IQR are updated and eventually the outliers could be considered as normal if they are after the update in the correct range.

Let consider the Figure 10. We can see that the 4 extreme values on the right are flagged as outliers and thus are not used to compute IQR, Q1, etc.

Using this approach, we will smooth the quality metric and discard inaccurate measures. Moreover, we are also re- 
4

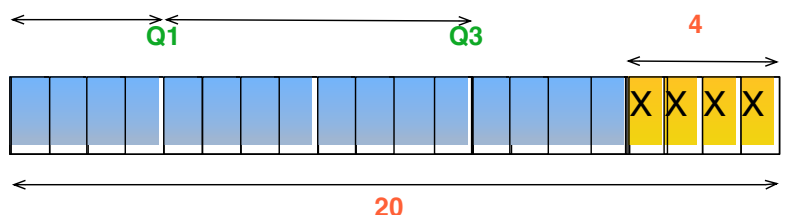

20

Figure 10: Sliding window example with 4 values flagged as outliers

active: we detect efficiently changing radio links and update accordingly their associated quality metric.

The reader can note that such a statistical approach could be easily applied to any metric measuring the quality of a radio link.

\section{RELATED WORK}

After theoretically working on protocol issues and energy savings in WSN, researchers have concentrated their efforts on experimentally studying the behavior of WSNs in the last few years $[18,19]$. Indeed, simulations are often too simplistic and new problems can arise in experiments that would have not been appeared in simulations. Barren et al. provide guidelines to conceive WSN testbeds [6].

Since experiments require much effort, some platforms aim at simplifying the performance evaluation of protocols and algorithms. Orbitlab [5] deployed a grid of sensors in an isolated environment. Some other approaches have been conducted for controlling more finely a testbed and obtaining reproducible experiments $[20,21,22]$. Lei et al. proposed to map real environments into an artificial testbed [23]. All these approaches aim at simplifying the deployment by proposing a controlled environment.

Other approaches aim at deploying an ad hoc testbed for evaluating the performances of one particular protocol, e.g. [5] for AODV and OLSR, [24] for network coding, [25] for a routing metric, etc. Their purpose is specific and researchers have hard time trying to generalize these results.

Recently, Raman et al. studied the problem of interference in IEEE 802.11 wireless mesh networks and tried to find out if the concept of a radio link is valid in this kind of networks [26]. Their results are experimental, but lead to fundamental and generic results in wireless mesh networks. Although it is focused on mesh networks, it gives an overview of what concerns may raise in Wireless Sensor Networks.

Some authors conducted real-world experiments. In particular, Barren et al. deployed a WSN to obtain meteorological data [27]. These measures will have been later used to derive meteorological models and to predict floods.

Ceriotti et al. deployed a WSN for measuring the movements of the Torre Aquila [28]. The authors provided feedback on the tools an engineer should deploy to design an efficient WSN. Both articles demonstrated that precise measures sometimes cannot be obtained from a single point of measurement. Distributed systems are more robust and permit to obtain rich information. However, they do not provide insights into the WSN environment itself. Thus, we proposed here to fill in this gap.

\section{CONCLUSION \& PERSPECTIVES}

In this paper, we have proposed a way to extend the use and the contribution of implemented testbeds in a urban environment. We have carried out thorough statistical analysis on a collected dataset to obtain an insight on the WSN environment and to emphasize its most distinguished properties.

Our analysis considered the aspects of WSNs such as the link characterization, correlation with environmental parameters (temperature, humidity, and luminosity) as well as network dynamics.

First, we showed that, contrary to the literature, there were no unidirectional links in our observed testbed, and more over that all bidirectional links are highly symmetrical while comparing their mean RSSI values. Furthermore, we have shown that RSSI values don't follow any of basic distribution models (Normal-Gaussian, Logistic and Cauchy): an accurate distribution still has to be proposed.

Although it is well-known that high humidity may cause decrease in link quality, we have showed that there is no mutual correlation between humidity and RSSI in our experiments. Even extreme maximum values of humidity don't cause significant changes in link quality measurements. This is most probably thanks to the MAC and $\mathrm{PHY}$ layers present in the radio chips.

We have highlighted that a proactive approach in neighborhood discovery may cause imprecise routing decisions, favoring the reactive solutions. Besides, although the RSSI exhibits large variations and is not correlated to the link quality, we were able to characterize the stable links. In particular, high RSSI (more than $-75 \mathrm{dBm}$ ) or a combination of both distance less than $70 \mathrm{~m}$ and RSSI between -75 and $-90 \mathrm{dBm}$ permit to conclude that we face to stable links.

Finally, we presented dynamic, reactive but still flexible mechanism for detecting and discarding transitory outlier values in measured RSSI value.

Besides, we could now give some advices to researchers who aim at deploying a testbed for characterizing the WSN environments:

- the network should be globally synchronized: we would have been able to compute also average delays, and give upper bounds on delays;

- the Packet Error Rate should be included for both neighborhood discovery packets and data packets in order to obtain a second metric of reliability;

- Neighborhood discovery should gather the RSSI in both directions. In this way, the originator node will have instant bidirectional knowledge of the link quality indicator between it and all of its neighboring nodes. We would have been able to capture a more time varying property.

\section{ACKNOWLEDGMENTS}

The authors would like to thank the personnel of Orange Labs (especially Dominique Barthel \& Giyyarpuram Madhusudan) for providing the experimental data and some precious advices for exploiting it.

This work was supported in part by the French Government and the Competitive Clusters Minalogic and System@tic under the contract FUI SensCity. 


\section{REFERENCES}

[1] J.N. Al-Karaki and A.E. Kamal. Routing techniques in wireless sensor networks: a survey. IEEE Wireless Communication, 11(6):6 - 28, December 2004.

[2] Giuseppe Anastasi, Marco Conti, Mario Di Francesco, and Andrea Passarella. Energy conservation in wireless sensor networks: A survey. Ad Hoc Networks, 7(3):537-568, 2009.

[3] David Cavin, Yoav Sasson, and Andre Schiper. On the accuracy of manet simulators. In POMC, pages 38-43, Toulouse, France, October 2002. ACM.

[4] Mineo Takai, Jay Martin, and Rajive Bagrodia. Effects of wireless physical layer modeling in mobile ad hoc networks. In MobiHoc, pages 87-94. ACM, Long Beach, CA, USA, 2001.

[5] D. Rastogi, S. Ganu, Y. Zhang, W. Trappe, and C. Graff. A comparative study of aodv and olsr on the orbit tesbed. In Milcom, Orlando, Florida, USA, October 2007. IEEE.

[6] Guillermo Barrenetxea, François Ingelrest, Gunnar Schaefer, and Martin Vetterli. The hitchhiker's guide to successful wireless sensor network deployments. In Sensys, pages 43-56, Raleigh, USA, November 2008. ACM.

[7] Anukool Lakhina, Konstantina Papagiannaki, Mark Crovella, Christophe Diot, Eric D. Kolaczyk, and Nina Taft. Structural analysis of network traffic flows. In SIGMETRICS, pages 61-72, New York, NY, USA, 2004. ACM.

[8] Haakon Ringberg, Augustin Soule, Jennifer Rexford, and Christophe Diot. Sensitivity of pca for traffic anomaly detection. SIGMETRICS Performance Evaluatation Review, 35(1):109-120, 2007.

[9] Thomas Watteyne, David Simplot-Ryl, Isabelle Augé-Blum, and Mischa Dohler. On using virtual coordinates for routing in the context of wireless sensor networks. PIMRC, 2007.

[10] http://www.wavenis-osa.org.

[11] Thomas Watteyne, Isabelle Augé-Blum, Mischa Dohler, Stéphane Ubéda, and Dominique Barthel. Centroid virtual coordinates - a novel near-shortest path routing paradigm. Computer Networks., 53(10):1697-1711, 2009.

[12] Tereus Scott, Kui Wu, and Daniel Hoffman. Radio propagation patterns in wireless sensor networks: new experimental results. In $I W C M C$, pages $857-862$, Vancouver, British Columbia, Canada, 2006. ACM.

[13] R.P. Liu, Z. Rosberg, I.B. Collings, C. Wilson, A.Y. Dong, and S. Jha. Overcoming radio link asymmetry in wireless sensor networks. In PIMRC, pages 1-5. IEEE, 2008.

[14] Lifeng Sang, Anish Arora, and Hongwei Zhang. On link asymmetry and one-way estimation in wireless sensor networks. ACM Transactions on Sensor Networks, 6(2):1-25, February 2010.

[15] Kannan Srinivasan and Philip Levis. RSSI is under appreciated. In EmNets, Cambridge, MA, USA, May 2006.

[16] Peter. J. Huber. Robust Statistics. Wiley, 2004.

[17] K. Kaemarungsi. Distribution of wlan received signal strength indication for indoor location determination. In $I S W P C$, Phuket, Thailand, January 2006. IEEE.
[18] Adel Aziz, Alaeddine El Fawal, Jean-Yves Le Boudec, and Patrick Thiran. Aziala-net: Deploying a scalable multi-hop wireless testbed platform for research purposes. In MobiHoc S3, New Orleans, USA, May 2009. ACM.

[19] R. Riggio, D. Miorandi, I. Chlamtac, N. Scalabrino, E. Gregori, F. Granelli, and Yuguang Fang. Hardware and software solutions for wireless mesh network testbeds. IEEE Communications Magazine, 46(6):156-162, June 2008.

[20] Juan Flynn, Hitesh Tewari, and Donal O'Mahony. A real-time emulation system for ad hoc networks. In $C N D S$, pages 115-120, San Antonio, USA, January 2002.

[21] James T. Kaba and Douglas R. Raichle. Testbed on a desktop: strategies and techniques to support multi-hop manet routing protocol development. In MOBIHOC, pages 164-172, Long Beach, USA, October 2001. ACM.

[22] Sagar Sanghani, Timothy X. Brown, Shweta Bhandare, and Sheetalkumar Doshi. EWANT: The emulated wireless ad hoc network testbed. In WCNC, New Orleans, USA, March 2003. IEEE.

[23] J. Lei, R. Yates, L. Greenstein, and Hang Liu. Mapping link snrs of real-world wireless networks onto an indoor testbed. IEEE Transactions on Wireless Communications, 8(1):157-165, January 2009.

[24] Sachin Katti, Hariharan Rahul, Wenjun Hu, Dina Katabi, Muriel Medard, and Jon Crowcroft. Xors in the air: Practical wireless network coding. IEEE/ACM Transactions on Networking, 16(3):497-510, June 2008.

[25] John Bicket, Daniel Aguayo, Sanjit Biswas, and Robert Morris. Architecture and evaluation of an unplanned 802.11b mesh network. In MOBICOM, Cologne, Germany, August 2005. ACM.

[26] Bhaskaran Raman, Kameswari Chebrolu, Dattatraya Gokhale, and Sayandeep Sen. On the feasibility of the link abstraction in wireless mesh networks. IEEE Transactions on Networking, 17(2):528-541, April 2009.

[27] G. Barrenetxea, F. Ingelrest, G. Schaefer, M. Vetterli, O. Couach, and M. Parlange. Sensorscope: Out-of-the-box environmental monitoring. In IPSN, St. Louis, Missouri, USA, April 2008. IEEE/ACM.

[28] Matteo Ceriotti, Luca Mottola, Gian Pietro Picco, Amy L. Murphy, Stefan Guna, Michele Corra, Matteo Pozzi, Daniele Zonta, and Paolo Zanon. Monitoring heritage buildings with wireless sensor networks: The torre aquila deployment. In IPSN, San Francisco, USA, April 2009. ACM/IEEE. 